bacilli were found vary in different individuals belonging to the same pregnancy from 2 in one to 26 in another. Not a single tubercle bacillus was found by microscopical examination of the mucous membrane of the uterus or the vagina. In the open lumen of the uterus twice only an isolated bacillus was detected in close neighbourhood of the embryo. Of course no pathological changes of the maternal organs due to the tubercle bacillus could be expected in so short a period, but there was every reason to expect numerous tubercle bacilli in the open lumen of the uterus, or, at least, adjacent to the mucous membranes of uterus and vagina. This, however, is not the case, and it seems, strange as it sounds, that all bacilli which do not make their way into the ova are eliminated from the genital tract. The mode by which the bacilli reach the female cell has not yet been dis. covered. To decide the question whether tubercle bacilli can be carried into the ovum by the spermatozoon, experiments are in operation at present with Ascaris megalocephala. But for the decision of the general question it is irrelevant whether bacilli introduced together with the semen reach the ovum at the very moment of impregnation, or shortly before or after it.

Further experiments will have to demonstrate how the bacilli develop in the embryo, at what time the first anatomical and histological signs of tuberculosis become apparent, and whother the mother remains healthy, or becomes infected in her turn (choc en retour). It will also have to be shown in embryos at the earliest stages before the formation of a placenta, that males rendered tuberculous by intraperitoneal, intratracheal, intraocular, etc., injection produce infected offspring, while the mother remains immune. It may be added that experiments in which tubercle bacilli were introduced into the bladder by way of catheter have given negative results. The same result followed after the injection into the vagina of the semen of fresh killed males, which had been previously mixed with a culture of tubercle bacilli. The author is aware that his mode of experimental procedure does not correspond with the natural course of things, in particular that, although one or two drops of a very thin emulsion were injected, the sperma contained a great many more bacilli than are ever to be found in the semen of a phthisical patient. Yet these experiments seem to prove that tubercle bacilli introduced into the vagina, together with the semen, may pass into the embryo without the intermediary of a mother. Of course it is a question altogether different how frequently, if at all, this takes place in human tuberculosis. On this point we know nothing.

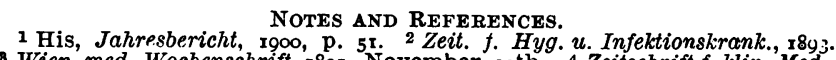
Wien. med. Wochenschrift, r89x, November 20 th. 4 Zeitschrift f. klin. Med. xiii, rgor. These experiments have been made under the supervision of Professor Gerbard, the well-known teacher of clinical medicine. Pro等 remarked that experiments made on ouinea-pigs or berlin. remarked that experines rats, and white mice hover the semen, a viscous thick matter, which introduced into the vagin congeals into a hard plug, completely flllng up the vagina up to the uterus. The general opinion that this plug prevents the outflow of th removal of the plug for the introduction of the be correct, because afte take place.

\section{THE USE OF PARAFFIN}

FOR RESTORING THE BRIDGE OF THE NOSE. By STEPHEN PAGET, F.R.C.S.,

Surgeon to the West London Hospital; Surgeon to the Ear and Throat Department of the Middlesex Hospital.

IT seems likely that the subcutaneous injection of paraffin, after the method discovered by Gersuny, is a very great improvement on all other treatments in cases where the bridge of the nose is sunken by injury or disease. The simplicity of the method, and the excellent result that is at once produced, are in strong contrast with the difficult, tedioss, and uncertain course of plastic operations, and of all attempts to use metal, celluloid, and the like substances. There is indeed something almost absurd in the mere injection of the paraffin, and the immediate restoration of a fairly good profile to a face that has been unhandsome for years; and the novelty of the method may justify thiz note on two cases where it was used.
The first case was that of a student, whose nose had been badly broken by a fall when he was 7 years old. There was an old fracture of the septum, but no marked nasal obstruction and no lateral deviation of the nose. But the whole middle portion of the nose was sunken, and the lower portion was broad and ill shaped, with the nostrils circular and looking rather forward. Under an anaesthetic the septum was refractured and straightened, a Walsham's nasal splint was applied to it, and a small quantity-about 2 c.cm.-of paraffin was injected under the skin in the middle line.

The second case was that of a man about 30, suffering from laryngeal phthisis, whose nose was sunken from disease, probably syphilitic, many years ago. In this case the interior of the nose was left untouched, and, under an anaesthetic, about $4 \mathrm{c} . \mathrm{cm}$. of paraffin was injected under the skin.

The result in both cases was very satisfactory ; the profile is restored, and the patient is pleased at his improved looks. Of course, in cases of this kind, there are faults that cannot be corrected either by Gersuny's method or by any other. A nose that is too short cannot be lengthened, and a nose that is too broad across the nostrils cannot be diminished in breadth. But the advantage of the paraffin treatment is that it accomplishes, in a few minutes, without a scar, more than plastic surgery can ensure by a severe operation. With a needle and a syringe and a bottle of paraffin, the surgeon can, in five minutes, remedy that sunken deformity of the nose which is so distressing to a man, not only on account of its unsightliness, but because it is taken for granted as positive evidence of syphilitic disease.

In the first case, a paraffin (Eckstein's) was used, that had a melting-point of $136^{\circ} \mathrm{F}$. In the second, a paraffin with a melting-point of $115^{\circ}$. The following considerations are to be noted :

1. Whatever may be the melting point of the paraffin, it must be kept, during use, $10^{\circ}$ or $15^{\circ}$ higher, or it will solidify in the needle before it can be injected.

2. For the same reason, the needle and syringe must be kept $15^{\circ}$ or $20^{\circ}$ higher than the paraffin. The loss of heat from the syringe may be to some extent checked by casing it in a bit of drainage tube.

3. An ordinary glass antitoxin syringe, with a well-fitting asbestos piston, answers every purpose. The needle must be broad and strong, such as is used for exploring the pleural cavity; but the needle generally used for this purpose is too long, and should be shortened to an inch and a half.

4. An assistant must make firm pressure, very carefully, all round the nose, and must keep up this pressure till the paraffin is set. But it sets almost at once, allowing only a quarter or half of a minute to the surgeon to mould it. The firm pressure may be helped by the use of a strip of lead or pewter under the tips of the fingers.

5. The skin of the nose, at the point where the needle is to enter, should be just nicked with a scalpel, so that the needle may pass easily. It is best to direct the needle downward, away from the eyelids, and to introduce it at the middle line of the nose.

6. Eckstein's paraffin, melting at $136^{\circ}$, is difficult to use, and must be very quickly transferred from the bottle into the subcutaneous tissue. Probably it is best suited for a case where only a very small quantity of paraffin is required. With this paraffin the syringe must be kept so hot that it can hardly. be handled except with gloves.

7. One case has been recorded of sloughing of the skin, presumably from heat. This disaster coula hardly happen with paraffin melting at $115^{\circ}$. More than one case has been recorded where signs attributable to pulmonary embolism followed the injection. It is therefore necessary to avoid piercing a vein, and to keep firm and close pressure all round the nose during and after the injection.

8. After the treatment a fold of lint should be kept over the upper part of the face, and kept moist with cold or iced lotion. In the two cases quoted there was little or no pain after the operation, but some swelling round the nose for three or four days.

It is, of course, impossible by any method to make a narrow or refined bridge to the nose in these eases. The front view of it is still heavy and clumsy, but the profile view is excellent, and this good result is obtained without any scar and without any severe or difficult operation, in a fow 
minutes. The paraffins can be procured frcm Mr. Rogers, 327, Oxford Street. A paraffin has been used in some cases that has a melting point of $104^{\circ}$ only, but there is some reason for thinking that this melting point is too low to give a permanent good result.

I am, of course, indebted to Dr. Scanes Spicer for his account of this method. He was, I believe, the first to use it in this country. In the second of my cases the result is not all that it ought to be; there is a fullness to one side of the middle line; but the profile is good. In the first case the result is absolutely satisfactory.

\section{MEMORA NDA: \\ MEDICAL, SURGICAL, OBSTETRICAL, THERA- PEUTICAL, PATHOLOGICAL, ETc.}

\section{NOTE ON A CASE OF MAMMARY ABSCESS FOLLOWING TYPHOID FEVER.}

IN the Gulstonian Lectures for 1900 Horton-Smith says : Speaking generally, it will be true to say that while it is very common to bone, in suppurations occurring elsewhere its presence is undoubtedly

And McCrae, ${ }^{1}$ in an article on Mastitis in Typhoid Fever, expresses the opinion that

Mastitis is apparently one of the rarest complications of typhoid fever, and occurs much less frequently than orchitis, thyroiditis, or parotitis.

He gives notes of 3 cases of his own, and refers to several cases recorded by other observers, but in only 2 was the bacillus typhosus isolated. ${ }^{2}$ In recent literature there have been published cases in which the typhoid bacillus has been recovered from the discharge in subdiaphragmatic abscess ${ }^{3}$ suppurative epididymitis, ${ }^{4}$ suppurative orchitis, ${ }^{6}$ cholecystitis, ${ }^{8} 7^{73}$, abscess of thyroid and orchitis, ${ }^{8}$ abscess in rectus abdominis, ${ }^{910}$ abscess in spleen, ${ }^{10}$ empyema, ${ }^{11}$ suppurating ovarian cyst, ${ }^{12}$ and axillary abscess, ${ }^{13}$ but unfortunately it has not always been stated whether or not the bacillus was present in pure eulture. It would seem, then, that the following case possesses sufficient interest to be worthy of record. For the history and material I am indebted to Dr. Edwin Bates of Wimbledon, who writes:

M. M., female, aged ${ }_{38}$, unmarried, was admitted into the Monsall Fever Hospital in August, 190r, suffering with enteric fever. She was there for ten weeks and then went to a convalescent home. During her stay in the home she was in bed for eight wceks with a painful swelling in her kneejoint. The pain and swelling subsided under rest and hot fomentations. At the beginning of February, 1902, she developed in the right breast a small painful swelling, which increased in size and finally burst. This abscess was scraped, and some of the discharge sent to the Institute on March 14 th, 1902 .

The discharge was a thin blood-stained seropurulent fluid which microscopical examination showed to be full of bacilli. In a dilution $\mathrm{I}$ in 30 it gave a complete agglutinative reaction with the laboratory typhoid culture. Gelatine plates made from the discharge yielded a pure culture of a bacillus which gave all the cultural reactions of the bacillus typhosus. A guinea-pig, inoculated with a 48-hour broth culture of this organism, died in 24 hours. The bacillus was recovered from the spleen, and acted towards enteric blood just in the same way as the laboratory stock culture.

On March 27th Dr. Bates kindly sent me some more of the discharge and $a$ specimen of the patient's blood. The blood, in a dilution 1 in 30 had only a partial effect upon the bacillus isolated from the discharge. The discharge itself, on the other hand, in similar dilution, gave a complete seruma reaction with both the bacillus isolated from the abscess, and the stock bacillus typhosus. Unfortunately no information could be obtained as to the previous agglutinative power of the blood.

Jenner Institute.

\section{Alfred MadConiex.}

REFERENCES.

1McCrae, Johns Hopkins Hospital Bulletin, vol. xiii, No.r30, p. $20.7^{2}$ Fornaca, and Davis Patterson and Hewlett. 3 Caton and Thelwall Thomas, BRITHE MEDICAL JOURNAL, 1900, vol. ii, p. 1790. 4 Strasburger, Mïnch.med. Woch., 1899. ${ }^{5}$ Lannois, Sem. Méd., rxoo, p. 177. 6 Droba, Centralbl. f. Bakt., 'xoo. '7 Parmentier, Sem. Méd., 1900, No. 25. 8 Murray, Philad. Med.

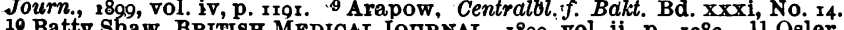

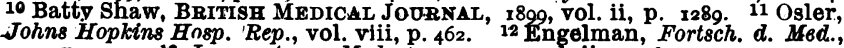
sgor, p. 1000. ${ }^{13}$ Journ. Amer. Med. As80c., r900, vol. ii, p. 36.
CANNABIS INDICA IN CHOREA AND PERTUSSIS. EARLY this year I was called to a severe case of chorea. The patient, a bright girl of 11 , was suffering from her third attack in five years. I began to give increasing doses of Fowler's solution with apparent success, but a fortnight later she was frightened by a steam fire-engine dashing past the house at night, and the next morning I found her flinging her arms and legs about with such violence that they were severely bruised against the back and legs of the couch on which she lay. Fearing death from exhaustion I put her on opium, of which 3 to $4 \mathrm{gr}$. a day was required. Then the appetite failed, and she had, in addition, a severe attack of toothache necessitating the removal of an upper molar under gas, which was administered only with great difficulty. This operation again intensified the movements, and I resorted to Indian hemp giving her $7 \frac{1}{2}$ minims of the tincture made from Parke, Davis and Company's fluid extract every four hours. The effect was magical. Next morning she was lying composed on the couch, looking perfectly happy, with only slight muscular twitching, and her appetite was improving. In three weeks she could dress and feed herself without much assistance, and has now been, for over two months, strong and well.

While attending this case I was called to one of the pertussis in a boy of 22 months. The cough was almost incessant, accompanied by vomiting. I gave him two minims of the tincture of cannabis indica every three hours. The next day I was told he had a very good night, coughing only three times. On the third day the cough had almost ceased, and by the end of the week had disappeared. In a second case, shortly after, that of a girl aged 5 years, I gave five-minim doses every four hours. After the second $4 \mathrm{oz}$. mixture had been finished the mother told me that the girl only coughed slightly about once a day. She took no more medicine and is quite well.

In all three cases the following points were noticeable: (I) Direct relief to spasm; (2) absence of any head symptoms; (3) non-interference with appetite or digestion.

I may add that only a little chloroform was added to the tincture to conceal the slightly unpleasant taste, and that the mixture was well taken in each case.

Birmingham.

E. T. Burton, L.S.A.

COMPLETE INTESTINAL OBSTRUCTION BY GALL STONE AND A BAND.

ON December 4 th, I90I, I was called to a lady, aged $7 \mathrm{I}$, who suffered from periodical attacks of abdominal pain. She told me that on going to bed the previous evening she was seized with sharp pain in the umbilical region, and quickly vomited bilious matter ; both had continued. A dose of castor oil had been taken and returned without affecting the bowels.

Upon examination I found a little abdominal distension and slight tenderness. Her temperature was $97^{\circ}$, and pulse 100 . I had her fed per rectum, and various attempts were made by oil, turpentine, and soap enemas to relieve the bowels, but these failed. A hypodermic injection of morphine and atropine relieved the pain.

On December 7 th the vomit became faecal, and Dr. H. J. Thornton saw her twice in consultation with me. Her condition was practically as above stated; on deep pressure about 2 in below the umbilicus to the left side, a slight resistance was felt, and a little pain elicited. Unless relieved by Nature, we advised the friends that the only chance was an abdominal section, but did not press it, because of old mitral trouble.

On December 8 th, neither faeces nor flatus having passed, the patient and her friends consenting, I made an abdominal section, with the help of Drs. Landon and Thornton.

Haying made an incision in the middle line below the umbilicus of sufficient size, a hand was passed into the abdo: minal cavity, and search made for the obstruction. A band of omentum on the left side was found, attached at its lower end to the upper and anterior margin of the pelvis, whilst its upper end was fixed near to the splenic flexure of the colon. Between this band and the abdominal wall several coils of intestine had passed, and that portion of gut occupying the lower angle thus formed was found distended on one side, on the other perfectly empty. The incision having been enlarged, and the gut drawn out, it was found to contain a large gall stone, which evidently had passed along it until it came to the band, where it formed a complete obstruction. I made an 